\title{
Bibliothekarische Kataloge als Spiegel und Instrumente von Wissensordnungen in der Frühen Neuzeit
}

\section{Systematisierung von Wissen}

Das Thema meines Aufsatzes werde ich nicht in historisch-demonstrativer Weise angehen - der Titel sagt ja bereits deutlich etwas über seinen Inhalt aus sondern vielmehr versuchen, auf spekulativem und theoretischem Weg voranzugehen, in der Hoffnung, Prinzipien und Modelle von einiger Tragweite ausmachen zu können.

Es gilt nicht nur festzuhalten, dass die beste Interpretation einer historischen Epoche diejenige ist, welche diese in eine allgemeine explikative Theorie einschließt; ich bin vielmehr überzeugt, dass die Notwendigkeit eines Erforschens und Verstehens der Bibliotheken sich nicht auf die Institutionen der Vergangenheit beschränken darf, sondern sich auch auf die gegenwärtigen erstrecken muss, nach Möglichkeit in einem gemeinsamen hermeneutischen Rahmen.

Mit Sicherheit dürfen sich in diesem Fall, wie in vielen anderen auch, die Analyse und historische Rekonstruktion nicht auf die Anwendung einer Interpretation ad hoc reduzieren, sondern sie sind gehalten, nach Erklärungen zu suchen, die für die Erhellung und Durchdringung viel größerer und vielfältigerer Gegebenheiten gültig sind. Die Geschichte würde überflüssig, wenn sie nicht den Anlass für die vertiefte Kenntnis der Gegenwart böte, sie würde nutzlos, wenn sie sich beschränkte, den begrifflichen Schatten und die ideologische Schablone der Gegenwart auf die Vergangenheit zu beziehen.

Geschichte ist also nicht die Abstellkammer von Formen und Materialien, die auf passive Weise durch die Verfahren einer blinden Gelehrsamkeit evoziert werden, sondern sie ist das Gelände eines Experiments und ein Hypothesenlaboratorium für das heutige Leben. In diesem Sinn gilt nicht allein der Gemeinplatz `historia vitae magistra - womit keine Sammlung moralischer Exempla, sondern die Erfahrung und Wiederbelebung einer vergangenen Gegenwart existenziellen Realität gemeint ist - son- dern auch die Überzeugung, dass das Wissen als solches nur produziert wird, wenn es sich in seiner Aktualität erneuert.

Seit dem Beginn des Buchdrucks haben sich die Auswahl, die Sammlung und die Ordnung der Bücher als Probleme und Sorgen ganz neuer Art herausgestellt; seit den Zeiten der Kunei-Formen-Tafeln, seit den chinesischen Enzyklopädien und den Buchrollen der Bibliothek von Alexandria hat man kaum stärker das Bedürfnis verspürt, bibliothekarisches Rüstzeug zu schaffen und anzuwenden. $\mathrm{Zu}-$ gleich zeigte sich die Notwendigkeit, immaterielle Sammlungen aufzubauen, die - eben unter dem Namen der Bibliothecae - das Gedächtnis und die schriftliche Dokumentation dessen anboten, was publiziert wurde.

Während Wissenschaft, Literatur, Bildung und Lehren den intellektuellen Kosmos repräsentierten und die Buchproduktion den materiellen und kommerziellen Pol konstituierte, übernahmen die Kataloge und Bibliographien die Aufgabe, kognitive Instrumente für beide Realitäten anzubieten, Karten des Zugangs, der Erforschung und des Nachweises zur Verfügung zu stellen. Es kam zur Herausbildung von drei Realitäten, deren jede sich in einem eigenen System verkörperte und zugleich mit den anderen in Korrespondenz stand: Weil jede von ihnen auf der Ebene der schriftlichen Kommunikation, des wissenschaftlichen Fortschritts und des Buchhandels notwendigerweise die jeweils anderen implizierten. In der Folge kam es dazu, dass zwischen den drei Systemen begriffliche und andere Isomorphismen zur Geltung kamen, sowohl im Reich der Literatur als auch in dem der Kultur und der Systematik.

`Bibliothecae`, 'Specula‘, 'Systemata‘, 'Encyclopaediae`, >Pandectae`, >Polyantheae $<$ etc. konstituieren Einrichtungen zur Anwendung sowohl der Organisation der Erkenntnis als auch derjenigen der Bücher oder derjenigen der Nachrichten über Bücher; das beweist in der Tat die bibliographische 
Phänomenologie, von den bibliothekarischen Katalogen bis zu den Verlagskatalogen, von den gelehrten Katalogen und Bibliographien bis zu den Enzyklopädien. (In Bezug auf diese letzteren konsultiere man die Konstruktionen von Theodor Zwinger bzw. von Laurentius Beyerlinck mit unterschiedlicher und doch entsprechender Anordnung.) Indem Konrad Gessner seine eigene Bibliographie auf die publizierten Werke in den drei gelehrten Sprachen begrenzte, wollte er z. B. der Gefahr entgehen, dass die Überlieferung der zitierten Werke verloren ging; zu seiner Zeit war es sicher noch möglich, als Kriterium für eine intellektuelle Auswahl die sprachliche Unterscheidung zu wählen. In diesem Rahmen waren seine Bemühungen weniger theoretischer als vielmehr praktischer Natur, sie bestanden nämlich in der Anwendung eines unkritischen Verfahrens in einem homogenen kulturellen Horizont, dessen Ende von zwei nicht umstrittenen Richtungen markiert wurde, der christlichen Philosophie und dem klassisch-antiken Denken.

Wenn man daher nach theoretischen Begründungen suchte, wurden diese im Innern einer geltenden wissenschaftlichen Struktur gefunden, ohne dass man es als paradox empfand, dass Ordnungen als Funktion von Überordnungen aus dem Innern desselben begrifflichen Systems stammten. Nur eine vollständige Fusion zwischen der Ontologie, dem Glauben und der Sprache konnte annehmen lassen, dass es ein und dieselbe Logik gibt, welche alle Formen und Manifestationen des Seins durchdringen und regeln kann. $\mathrm{Da}$ nun einmal unabdingbar die Ordnung die Seele jedweder bibliothekarischen Sammlung ist - wie das für alle anderen komplexen Systeme gilt, vom Universum bis zu den lebenden Organismen - hatte Gessner keine Schwierigkeit, ausdrücklich zu erklären, dass die Kompetenz und die Legitimation der bibliothekarischen und bibliographischen Ordnungen zur Dialektik, und d.h. zur Logik, gehören. Und dennoch enthält nur der zweite von den drei bibliographischen Apparaten - nach Namen, Klassen und loci communes - d.h. die Pandectae, eine Sammlung und Aufstellung in kompletter und integraler Form sowohl der Elemente des Wissens als auch aller literarischen und textlichen Äußerungen, inklusive derer, die ohne die Vaterschaft eines Autors auftreten.

Auch Johann Heinrich Hottinger misst in seinem nach Sprachen gegliederten Bibliothecarius Quadripartitus (1664) der systematischen Katalogisierung - in sechs Klassen: Theologie, Jurisprudenz, Medizin, Historie, Philosophie, Philologie - der bibliothekarischen Organisation eine zentrale Bedeutung zu; dennoch beschränkt er sich nicht auf diese
Betonung der Anordnung, vielmehr taucht in der Konstruktion dieses Katalogs auch ein anderer, entscheidender und kritisch-fundamentaler Faktor für die Durchführung jenes Prozesses auf, >der aus dem Philosophen einen Bibliothekar macht .

Wenn die Philosophie den höchsten Grad des Wissens ausdrückt, heißt das im heutigen Verständnis, dass die Bibliographie, die nicht dazu gerechnet wird, ein höheres Stadium repräsentiert, in welchem die Struktur und die Anordnung der Verweisungen die Aufgabe haben, die Wissenschaft zu regulieren und zu regieren, ebenso wie ihre dokumentarischen Korrelate, d.h. die informative Substanz des verzeichneten Wissens. Nicht allein die Kataloge des 16. und 17. Jahrhunderts, sondern auch die Büchersammlungen selbst waren, wie man weiß, Einrichtungen >per artes et scientias rer Anordnung, in den logisch-methodisch festgefügten Folgen, finden sich die >res‘, weshalb die entsprechenden Kataloge auch entsprechende Bezeichnungen hatten: systematische Kataloge, Real-Kataloge, methodische, logische oder klassifizierende Kataloge. Die Nominal- oder Autorenkataloge hatten lediglich die Funktion von Verweisungen auf diese Kataloge.

Die Hinweise auf Gessner und Hottinger sollten hier kein anderes Ziel haben als die Herausarbeitung zweier wesentlicher Punkte: Einer besteht im friedlichen Gleichgewicht zwischen der noetischen und der bibliothekarischen Realität, welche beide dem universitären Disziplinenschema und der aristotelischen Logik untergeordnet waren; in den anderen drückte sich die Einsicht aus, dass die bibliographische Darstellung nicht nur eine Ableitung von jener Realität konstituierte, sondern auch eine Überschreitung, und d. h., dass sie sich auf einer logischen Metaebene in Bezug auf die Wissenschaft, die sie strukturieren sollten, befinden. Offensichtlich ist diese zweite Feststellung auf einer theoretischen und spekulativen Ebene am interessantesten. Um sie zu vertiefen und zu entwickeln, werde ich nun der Prüfung und der kritischen Diskussion Raum geben, die sich auf die Gesamtheit der Probleme bezieht, welche die Prozesse der schriftlichen Kommunikation charakterisieren, insbesondere solche, die um die Benutzung von Logiken und von Verfahren der Indexierung, Archivierung, Heuristik oder Repertorienanlage wuchern. Auf diese Weise wird die Ausführung des im Titel genannten Themas über die bloße ideologisch-historiographische Absicht hinausgehen und versuchen, in den Rahmen abstrakter und allgemeiner sowie derjenigen Interpretationen einzutreten, welche die Probleme der bibliographischen Modellbildung ansprechen, indem sie auf die entsprechenden spezifischen Ebe- 
nen der intrinsischen logischen und ontologischen Zusammenhänge verweisen.

\section{Bibliographien und Kataloge als Wissensordnungen}

Damit ein großer Wissensvorrat nützlich werden kann, muss er letztlich auf angemessene Art und Weise erschlossen und geordnet sein. Wenn auf neuronaler Ebene die kognitiven, erinnerten und auffindenden Prozesse prädisponiert sind und automatisch von bestimmten zerebralen Zentren ausgeübt werden, wenn es sich um im Gedächtnis oder in schriftlichen Quellen vorhandene Begriffe handelt, muss ihre distributive und geordnete Struktur von Grund auf in einem meta-noetischen Verfahren aufgebaut werden, damit das Wissen abgerufen werden kann, einzeln, oder in unterschiedlicher Zusammenstellung wie ein eigenes spezifisches Objekt der Analyse, der Organisation und der Erforschung. Die Notwendigkeit, Begriffe und Konzepte in einem meta-noetischen System zu strukturieren, hat seit den Zeiten oraler Kultur bestanden und sich z. B. in der Einführung von Hierarchien und genetischen Prozessen in Bezug auf mythologische und theogonische Gegenwarten und Ereignisse manifestiert, je nach Maßgabe eines Achsensystems, das über Tausende von Jahren zu den genetisch-chronologischen Vorstellungen gehörte.

Das Bewusstsein der meta-wissenschaftlichen Natur des Wissens, welches die Erkenntnis betrifft und insbesondere - das ist für unser heutiges Interesse wichtig - der Erkenntnisse, welche sich mit der Systematisierung sowohl der Ideen als auch von deren schriftlichen Zeugnissen beschäftigen, ist wenig anerkannt und deswegen kaum verbreitet. Sie wurden auf zwei Weisen untersucht: Eine ist die allgemeine oder systematische Epistemologie derjenigen wissenschaftlichen Erkenntnisse, die mit dem 20. Jahrhundert in Vergessenheit geraten sind; die andere die Disziplin der Klassifikation von Büchern, welche aus der Notwendigkeit erwuchs, eine Einrichtung für die bibliothekarische und informationale Organisation zur Verfügung zu stellen, die sich heute darauf beschränkt sieht, Schemata für die bibliothekarische Verzeichnung und den Nachweis (etwa durch Dezimalklassifikation) anzubieten. Wenn die gemeinsame Behandlung der ideologischen und der dokumentarischen Gegenstände tatsächlich verfolgt werden kann - was man durch Jahrhunderte hindurch etwa in der Konstruktion von Klassifikationen von Büchern tat - in all jenen Fällen, in denen jedem Bereich, jedem Thema oder jedem wissenschaftlichen Problem ein Dokument entsprach, das darüber vollständig und erschöpfend handelte, so blieb dennoch das Problem ungelöst, die Ergebnisse in ein planbares und kohärentes System zu integrieren. Noch schwerer wiegt aber das andere Problem, das sich auftut, wenn die Entsprechungen zwischen Gegenständen und Dokumenten nicht parallel und erschöpfend sind.

Zunächst einmal muss man aber die grundlegende Verwechslung klären, die in deutsch- wie englischsprachigen Ländern die Entwicklung kritischer Untersuchungsmethoden innerhalb der Substanz katalographischer Realität behindert, insbesondere mit Rücksicht auf die Realitäten, die durch eine semantische Natur bezeichnet oder determiniert sind. Letztere besitzen theoretische Knoten und praktische Schwierigkeiten, die direkt mit der Organisation jener intellektuellen Sphäre verbunden sind, welche den strukturellen Ort für einzelne Kategorien und Begriffe konstituiert.

Während die Anwendung von nominalen und textuellen Katalogen sich als ein - selbsttrügerisches - Derivat der gelehrten und literarischen Disziplinen darstellt, woraus im Übrigen die fatale Irrelevanz der Bibliographie heute resultiert, erfordert die Errichtung von semantischen Katalogen, dass man nicht nur über Theorien, sondern auch über Wegweiser, Karten und adäquate epistemologische Hilfsmittel verfügt. Diese erweisen sich allerdings, sowohl auf der Ebene der Ideation als auch auf der der Konstruktion, als derartig komplex, dass sie noch heute großenteils nur in einem Stadium angetroffen werden können, welches ihrer späteren Individuierung vorangeht.

Negativen Einfluss auf das Studium und die kritische Analyse der Kataloge hat auch die Meinung, nach welcher die Bereitstellung von Katalogen als Instrumente des Zugangs zu den Büchern einer Sammlung sich auf Grund rein technischer Prozesse vollzieht, für deren Durchführung die Kompetenzen der Vertreter der physischen und logistischen Verwaltung einer Bibliothek ausreichten.

Ein analoges, nicht weniger verheerendes Urteil läuft darauf hinaus, dass die Bibliographie allgemein eingeschränkt und reduziert wird auf einen Verweisungs- und Evidenzierungskomplex, dessen Aufgabe es sei, je nach Thema und Gegenstand der Forschung die entsprechenden Sedimentationen oder die älteren Werke verfügbar zu machen. Was in dieser Vorstellung verloren geht, ist die Fähigkeit, die Prozesse der bibliographischen und katalographischen Vermittlung zu gewichten und in Begriffe zu fassen, die, zunächst investigativ und dann operativ, jede grundlegende epistemologische Bedingung charakterisieren, die das gesamte System der Archivierung und der entsprechenden Katalogisie- 
rung der gespeicherten Kommunikation unterfüttern. Man zieht nicht mit der genügenden Aufmerksamkeit die Tatsache in Betracht, dass beide, Bibliographien und Kataloge, die Struktur von Indices haben, die ermöglichen, ontologische Einteilungen bzw. literarische Nischen oder Textsegmente der aufgezeichneten Erinnerungen zugänglich zu machen. Deren Organisation und Ausführungsverfahren, deren Methodologie und kritische Prüfung bilden eine disziplinäre Materie, welche sowohl die der Bibliographie als die der dazu gehörenden Katalogisierung ist. Die Indices sind Apparate des Verbindens und des Aufrufens, welche, in Modulen von dreiwegigen Beziehungen, die besonderen vermittelnden Funktionen zwischen den beiden Wirklichkeiten erklären, zwischen den Dokumenten und den Benutzernachfragen. Die Strukturen der Indices können freilich nicht funktionieren, wenn nicht eine passende logisch-semantische Architektur aufgebaut und organisiert wird, die mit geeigneten $\mathrm{Ka}$ tegorien ausgestattet ist, so dass diese die Realität der Dokumente wie die geistige Realität aufnehmen und zwischen ihnen fruchtbare Forschungsrichtungen angelegt werden können.

Wer auch immer unter die Oberfläche der Repertorien und Verzeichnisse der elf Bände meiner Storia della Bibliografia ${ }^{1}$ gedrungen ist, konnte feststellen, dass dieses Unternehmen von einer grundlegenden und zentralen Hypothese getragen wird, die man darin zusammenfassen kann, dass der rekonstruktive und hermeneutische Kern der Arbeit darin besteht, dass die Bibliographie in ihrer Rolle als Metadisziplin die Gesamtheit einer intellektuellen (linguistischen, textlichen, semantischen) Typologie repräsentiert, die mit einer Topographie des Inventars der entsprechenden dokumentarischen Objekte korrespondiert.

In dieser Vorstellung taucht zwar die Utopie der Historia Literaria ${ }^{2}$ wieder auf - nicht aber als Grabkammer des Abgelegten und träge Ansammlung von literarischen Objekten, deren Registrierung die intellektuelle Realität konstituierte, indem es sie verzeichnet -, sondern als notwendige Bedingung dafür, eine Sphäre der dynamischen Erinnerung und der intermedialen Kompensation zwischen dem aktiven Gedächtnis und der Erfassung zur Verfügung zu stellen. Dass diese Sphäre nicht allein sehr komplex ist, sondern auch in einem hohen Maße von logischer Multidimensionalität charakterisiert wird, kann nicht überraschen, angesichts der unvermeidlichen Subsistenz von dreiwegigen Beziehungen in jedwedem Prozess der Angabe geschriebener Quellen und der Präsenz einer höheren Zahl von abstrakten Welten der Verweisung, der Ideologie, der Sprache und der Texte. Anderes stand nicht zu erwarten, solange die für das Funktionieren des komplexen bibliographischen Systems nötige logische Potenz nicht auf eine höhere Ebene gehoben war, damit sie nicht allein die Welt der begrifflichen Substanzen und Formen umfasst, sondern auch die unendliche Vielfalt ihrer konkreten Manifestationen auf der Ebene der dokumentarischen Realität.

Während ich in meinem Buch über »das Wagnis der Bibliographie ${ }^{3}$ das Ungenügen der noch herrschenden Konzeption der bibliographischen Disziplin gebrandmarkt habe, beschränken sich hier die Weiterungen einer Konzeption über die eigentliche Natur der Bibliographie nicht auf die Beeinflussung des Wesens der traditionellen bibliographischen Disziplin, einschließlich der abgeleiteten Anwendungsverfahren, sondern sie verändern mit Sicherheit und grundlegend auch den Wert und die Funktionen der Geschichte der Bibliothek. Insbesondere diejenigen Konsequenzen sind bedeutsam, die eine korrekte Auslegung der Bibliographie für die Hermeneutik und die Ausrichtung dieser Disziplin haben können, wie ich das in einem Aufsatz von $1999^{4}$ zu zeigen versucht habe, der wenig später verändert wieder abgedruckt wurde. ${ }^{5}$

\section{Kultur und Struktur}

Wir haben bis hierhin die Existenz einer intellektuellen Welt und einer damit korrespondierenden dokumentarischen Phänomenologie betont; zum Abschluss dieses Diskurses gehört natürlich insbesondere die Erörterung ihrer Natur und ihrer Beziehungen.

Man sollte eher als von einer intellektuellen Welt von einer Gesamtheit intellektueller Welten sprechen, von denen es so viele gibt wie Zentren der informationalen Aufbereitung. Es ist sicher irrig und jedenfalls abwegig, sich vorzustellen, dass die soge-

1 Alfredo Serrai: Storia della Bibliografia. 11 Bde. Rom: Bulzoni 1988-2001.

2 Die "Historia Literaria« besaß zwei Defizite: Erstens gab sie vor, als Erbe einer entstellenden Bibliophilie, dass ihren Dokumenten der Rang höchster Wissenschaft und Kultur zukäme, und zweitens hatte sie kein Bewusstsein von ihrem eigenen ontologischen und metadisziplinären Status - wie im Übrigen auch die unmittelbar auf sie folgende Bibliographie.

3 Alfredo Serrai: Il cimento della bibliografia. Milano: S. Bonnard 2001.

4 Alfredo Serrai: Caratteri, insufficienze, ed ambiguità di Storia delle Biblioteche. In: Alfredo Serrai: Racemationes Bibliographicae. Rom: Bulzoni 1999, S. 9-44.

5 Alfredo Serrai: Storia delle Biblioteche. Intervento al Convegno su "La storia delle biblioteche", L' Aquila 16-17 Settembre 2002. In: Bibliotheca 3 (2003), S. 22-28. 
nannte Information etwas sei, was in einem anderen Ort als in einem lebenden und funktionierenden Nervensystem residieren könnte. Es ist notwendig, dass der Begriff der Information - abgesehen vom genauen, wenngleich engen Wert, den dieser Begriff in der Kommunikationstheorie von Shannon ${ }^{6}$ besitzt, wie auch abgesehen vom verallgemeinerten Wert der Nachricht, des Austauschs oder der sozialen Kommunikation - vor allem dazu eingesetzt wird, den Wert von sensitiven, psychischen, emotionalen und intellektuellen Reaktionen zu bezeichnen, die ein menschlicher Empfänger entwickelt, wenn er von äußeren oder inneren Anreizen berührt wird. Die Information darf nicht allein aus der Beziehung zum Inhalt der Dokumente verstanden werden, sondern - wenn wir uns z.B. in den Rahmen der alten, allgemeinen philosophischen Visionen der Renaissance versetzen, welche danach strebte, die natürliche Realität und diejenige des menschlichen Geistes in einem gemeinsamen göttlichen Ursprung zu vereinen - die Information wird als ein reaktiver und repräsentierender Prozess betrachtet, welcher die Gesamtheit der physischen und psychischen Objekte betrifft, vermittelt durch die Partizipation einer kognitiven Form der Wahrnehmung, der Empfindung, der Refiguration und der geistigen Konstruktion.

Ist einmal geklärt, was nicht unter Information $\mathrm{zu}$ verstehen ist, muss man auch erkennen, dass es so viele kulturelle Welten gibt, wie es Szenarien der zivilisierten Welt gibt, in denen sich einzelne individuelle Zentren bilden, leben und wirken; und dennoch darf die Gegenwart dieser weitläufigen Systeme einer kollektiven Matrix nicht den Status jener ontologisch primären Bedingung schwächen, nach welcher die tatsächlichen Wirkungsprozesse einzig und allein im Inneren einzelner Zentren der Wahrnehmung, der Verarbeitung und der kognitiven Operationalität ihren Ort haben. Es gibt nämlich keine Information, außer in der Form und der Wirklichkeit einer subjektiven Erkenntnis, modelliert und beeinflusst, wenn man will, von einer umgebenden und sozialen Kultur, so dass jedes Mal, wenn man von Information spricht, man diese notwendigerweise auf die Ebene, die Qualität und die Stufe der Aktualisierung heben muss, wie auch auf die aktuellen und konkreten Erfordernisse eines bestimmten Wissenssystems, welches als solches immer grundsätzlich individuell ist.

In der Welt der Kultur und der schriftlichen Überlieferungsreste erweist sich die Konfiguration der grundlegenden Verhältnisse als eine, die zwischen der Totalität der intellektuellen Welten und der Totalität der geschriebenen Zeugnisse stattfindet; und diese erscheint verteilt und gleichwohl in den Umständen und der Dialektik der Gegenwarten artikuliert, einerseits von aktiven Zentren der informationalen Rezeption, andererseits von der Existenz und Zugänglichkeit einschlägiger Büchersammlungen. Die Idee dieser doppelten Totalität ist dennoch nichts anderes als ein leerer Ausdruck, denn sie dient allein dazu, die abstrakte Existenz zweier Klassen von Entitäten zu beschreiben, ohne dabei die Oberfläche zu berühren oder wenigstens mit explikativen und operativen Modi in ihre Natur oder ihr Wesen einzudringen.

Wir wissen, dass beide zusammen nicht univok isomorph und keineswegs parallel sind, und dass sie sich nicht in einem Verhältnis strenger Korrespondenz befinden, sondern dass sie an sich höchstens Zeichen einer partialen Homologie oder gemeinsamer Beziehungen tragen, was weder für eine exakte Wahrnehmung ihrer Substanz genügt noch zur Präzisierung ihrer eventuellen korrelativen Beziehungen eigener oder reziproker Funktionalität.

Während die Gesamtheit der intellektuellen Welten - mit Ausnahme von relativ engen und beschränkten Kulturen wie etwa derjenigen des lateinischen Hochmittelalters - aus so vielen intellektuellen Welten zusammengesetzt ist, wie es ideologische Entwürfe und emotive Zentren einer Zivilisation gibt, ist die Gesamtheit der schriftlichen Zeugnisse ein Reflex, wenn auch unausgeglichen und partiell, nicht allein der rein geistigen Prozesse und Begriffe oder der wissenschaftlichen Eroberungen und literarischen Manifestationen, sondern, in einem merkbaren Teil, auch von informativen Sedimenten, administrativen Akten und patrimonialen Hinterlassenschaften in Nachfolge und als Spur von ökonomischen, sozialen, politischen, künstlerischen Aktivitäten etc. Eine Beziehung oder eine Verbindung zwischen beiden zu etablieren, ist nur innerhalb eines begrifflichen Rahmens möglich, der jedes einzelne Faktum von der dokumentarischen Phänomenologie her verbinden und rechtfertigen kann, insofern es auf die Ursachen und die Ereignisse der Kulturgeschichte, der Geschichte der Literatur, der Jurisprudenz, der Verwaltung, der Politik etc. bezogen wird. Dieser Rahmen ist weit gespannt und mit einer höheren logischen Komplexität sowohl gegenüber den einzelnen Segmenten des komplexen intellektuellen als auch des globalen dokumentarischen Systems ausgestattet, und identisch mit der Bibliographie, so dass wir uns auf ihn beziehen müssen, um die Natur und die Kompetenz dieser Metadisziplin zu bestimmen.

6 Vgl. Claude E. Shannon: Ein - Aus. Ausgewählte Schriften zur Kommunikations- und Nachrichtentheorie. Berlin: Brinkmann \& Bose 2000. 
Bisher wurde der Bibliographie die Aufgabe zugewiesen, die Gesamtheit des schriftlichen Erbes zu umfassen und zu ordnen, so dass die Charakterisierung, Erkenntnis und die Identifizierung der einzelnen Zeugnisse von der Zufälligkeit und Willkür bibliothekarischer Verwaltung behindert werden. Auch wenn Bibliotheken und Archive manchmal Zentren einer weiten und qualifizierten Sammlung von Dokumenten sind, repräsentieren die in ihnen zusammengetragenen Sammlungen höchstens bestimmte Ausschnitte und Aspekte der kulturellen Welt und dies im Hinblick auf einige ihrer Interpretationen. Ohne eine vereinheitlichende Karte und eine Beziehung zwischen den konkreten - selektiven, kontingenten und fragmentarischen - Bedeutungen der verschiedenen Büchergruppen wäre es nicht einmal möglich, eine grobe Topographie zu zeichnen.

Man könnte nun annehmen, dass diese integrative und rekonstruktive Funktion der Bibliographie mit steigendem Erfolg der wissenschaftlichen und literarischen Verantwortung einzelnen Teilen der intellektuellen Welt übertragen werden sollte, mit der Anforderung an die Gemeinschaft der Wissenschaftler, mit dokumentarischen Begriffen das Wissen ihres Bereichs in Gegenwart und Vergangenheit zu projizieren. Man vermutet überdies, dass eine solche Option den Vorteil hätte, unter anderem die kohärenten und strukturierten Ergebnisse sowohl im kognitiven wie im dokumentarischen Bereich auf der Ebene der Einheitlichkeit wie auf der der Globalität zu garantieren. Ein solches Verfahren ist gleichwohl unpraktikabel und daher offensichtlich unfruchtbar, und zwar aus drei Gründen. Der erste besteht in der fehlenden Gleichwertigkeit zwischen den Ergebnissen der historiographischen Entwicklung, sowohl der wissenschaftlichen wie der kritisch-ästhetischen, und den dokumentarischen Sedimenten: Wie ich anderwärts gezeigt habe, haben die logischen und informativen Dimensionen der bibliothekarischen Bestände eine Größenordnung, die über derjenigen der historiographischen Rekonstruktion liegt, die aus ihnen abstrahiert oder abgeleitet wird. Ganz zu schweigen davon, dass Historiker - für Gebildete und für Kritiker künstlerischer Produktion gilt das Gleiche -, wenn sie nicht zugleich Bibliographen wären, nicht in der Lage sind, die Fülle der original erhaltenen Bücher zu erfassen und zu überschauen.

Der zweite Grund besteht in der Feststellung, dass der gegenwärtige Stand der Wissenschaft und der Kultur das Ergebnis eines Versuches ist, der im Wesentlichen linear erscheint, der aber, um sich an gängige Wege anzupassen, alle anderen Wege außer Acht lässt und damit einen breiten Bereich verschie- dener Paradigmen, unwegsamer Strecken und, wie es nur natürlich ist, die Gesamtheit des korrespondierenden ideologischen Gepäcks und des entsprechenden schriftlichen Erbes.

Der dritte Grund besteht in der Beobachtung, dass die Wissenschaft heute sich immer mehr in globale epistemologische Visionen flüchtet, dass die philosophischen Konzeptionen es aufgegeben haben, metaphysische Systeme zu konstruieren, dass die Kultur sich in einem Magma von Theorien und Moden artikuliert, die schlecht verbunden und häufig widersprüchlich sind, dass die Verhaltensweisen und Tendenzen eine unbedeutende und tolerante Neutralität angenommen haben, dazu bestimmt, pragmatisch interpretative Lösungen, welche zwischen den verschiedenen Formen der Zivilisation ein Minimum an Spannung erzeugen, zu erreichen.

Wie auf logischer Ebene das Theorem der Unvollständigkeit von Gödel gezeigt hat, ist kein Kulturmodell in der Lage, eine adäquate und kohärente, d. h. von Widersprüchen freie Rechtfertigung der eigenen begrifflichen und axiologischen Struktur zu finden. Um diese zu erhalten, muss man auf immer stärkere Systeme zurückgreifen oder, anders gesagt, auf immer weitere, reichere und komplexere.

Um die Komplexität unseres kulturellen Systems $\mathrm{zu}$ verstehen, ist es also notwendig, es mit Absicht auf die Konstruktion immer stärkerer Systeme zu verlassen, die fähig wären, die Reichweite der eigenen konzeptuellen Kompetenzen zu verlängern, sei es durch Einbeziehung und Reflexion anderer Zivilisationen, sei es durch Ausdehnung der Grenzen und der Erkenntnisinstrumente selbst. Um Systeme größerer und wachsender Komplexität zu interpretieren, muss man fortschreitend abstraktere und gewagtere Formeln verwenden, die in der Lage sind, auf informativem Niveau die Öffnung von immer weiteren und tieferen Horizonten zu spiegeln. Daraus folgt, dass es unmöglich ist, überhaupt etwas in den eigentlichen spezifischen Begriffen des Sinns und des Werts zu erkennen, wenn sie nicht in einer funktionalen Beziehung mit anderen Dingen und in einem gemeinsamen Umfeld stehen, oder wenn sie nicht aus einer angenommenen und spezifischen Kategorie qualifiziert resultieren. Die Kategorien ihrerseits erhalten Kompetenz, Pertinenz, Maß und semantischen Ort in Beziehung auf andere Kategorien; diese zusammengenommen bilden ein Netz, welches sich in einen semantischen Raum einfügt oder ihn kreiert, der zugleich mit dem anderer Netze verbunden ist und logische Dimensionen aufweist, die sich nachfolgend steigern und immer höher steigen.

Die mitlaufende Präsenz der Rahmen, welche die verschiedenen und teilweise inkompatiblen Kultu- 
ren spiegeln, produziert nicht allein einen ideologischen und politischen Kontrast oder Widerstreit, sondern für die Betreffenden auch eine Unmöglichkeit, z. B. gemeinsame Kataloge aufzubauen. Kataloge sind unvermeidlicherweise jeder für sich Spiegel einer besonderen kulturellen Metaphysik: Wenn man Kataloge heterogener oder auswärtiger kultureller Provenienz übereinander legt oder vereinigen will, befindet man sich in der Situation, nicht mehr über diejenigen Hilfsmittel der Homogenität und der Kohärenz zu verfügen, welche die unabdingbare Bedingung für ihre Konstruktion und ihr korrektes und nützliches Funktionieren sind.

Wenn die Kataloge auf eine Weise eingerichtet sind, eine Kultur in den Gesetzmäßigkeiten einer anderen begreifen zu können, ergeben sich nicht allein theoretische Situationen voller Aporien oder Widersprüche, vielmehr erscheint vom Gesichtspunkt der Kultur aus, die marginalisiert oder geopfert wird, unmittelbar das Bewusstsein eines plötzlichen Unverständnisses, von Überraschungen und von Gewalt.

\section{Die Grenzen von Katalogen}

Die Anordnung einer Büchersammlung zu dem Zweck, einer spezifischen Benutzung zu genügen, gehört $\mathrm{zu}$ jenen Handlungen, die wegen vorgeblicher Leichtigkeit sich technisch oder banausisch nennen. Eine solche Anordnung, allgemein unter der Gattung der Kataloge, gibt sich unfreiwillig elementar, insoweit sie die normalerweise dreiwegige Bedingung, welche die Indizes charakterisiert; auf zwei Begriffe, den des Buches und den des Lesers, reduziert. So wird scheinbar sowohl die Bandbreite der dokumentarischen Fälle als auch die der Benutzerbedürfnisse vereinfacht.

Die dreiwegigen Beziehungen machen hier ihr Recht geltend und der daraus resultierende Kata$\log$, sei er nun eingerichtet in gegenläufiger Richtung Dokument-Benutzer und Benutzer-Dokument, besitzt keine definierbare Physiognomie mehr, keine Erkennbarkeit oder Antizipierbarkeit. Anders gesagt: Er ist ein Katalog, der allein in einem Zustand der Partialität und der substanziellen Ineffizienz funktionieren kann. Daraus folgt, dass, während die Bibliotheken Konnotationen und Bedeutungen erwerben, so lange es gelingt, ihre Konfiguration auf eine bibliographische Karte zu projizieren - in einer Weise, dass wenigstens Teile von ihr wieder erkannt und identifiziert werden können -, die dazugehörigen Kataloge, und insbesondere die semantischen, unvollständig und ineffizient bleiben, weil sie nicht im Stande sind, Legitimation aus einer homogenen und kohärenten bibliographischen Matrix zu beziehen, noch auch von einem sicher definierten und konstanten Benutzerprofil bestätigt und bewertet werden.

Trotz der oft wiederholten Behauptung, dass Kataloge die Benutzervorstellung einer Sammlung repräsentieren müssen, scheint mir, wenn diese in bibliographischen Begriffen konfigurierbar und formulierbar sind, folgt aus dem Gesagten die Unhaltbarkeit einer linearen Beziehung oder einer einfachen unmittelbaren Abhängigkeit zwischen einer Büchersammlung und den Katalogen, in denen sie erfasst sind. Die Kataloge sind Ausdruck einer Sammlung in Funktion einer ganzen Bandbreite von Benutzerkategorien. Auch wenn ihr informativer Inhalt ein Auszug der Indexierung von Daten ist, die in dieser Sammlung enthalten sind, muss die Auswahl, welche gemacht wird und die beratende Einrichtung, die sie zur Disposition stellt, in einer Funktionsbeziehung zu den Interessen und den Anforderungen der Leser stehen.

Das impliziert, dass - selbst in der Gegenwart einer Bibliothek mit erkennbarer bibliographischer Physiognomie, die anders weder wahrgenommen noch in bedeutsamer Modulation angezeigt werden könnte - die Kataloge, welche deren informative und literarische Substanz vermitteln, nicht eine einzige Abstraktion der Sammlung konstituieren, sondern dass sie Teil des Ganzen sind, welches in Begriffen des Interesses, der Begegnung, der Befriedigung, die effektivsten Beziehungen mit den konkreten, potenziellen oder idealen Benutzern der Bibliothek einrichten wird.

Wenn wir daher entweder eine absolute Vorrangigkeit der Sammlung vor der Begegnung mit den Lesern negieren (weil alle Sammlungen, selbst die bibliographisch vereinheitlichten, nicht weniger willkürlich als die Anforderungen eines angenommenen Lesers erscheinen oder eine bloße Äquivalenz zwischen Struktur der Sammlung und Einrichtung der entsprechenden Kataloge negieren), wer wird die Bedingungen jener Funktionalität präzisieren und individuieren, welche die Bibliotheken über den Umweg ihrer Kataloge aktiv und produktiv machen? Offensichtlich wiederum allein die Bibliographie.

Durch Informatik und Telekommunikation scheint die bibliothekarische Präsenz der Bücher wenigstens prinzipiell in dem Sinne, dass die Forscher noch zu einer Einführung in die Gelehrsamkeit genötigt sind, wofür die >Bibliotheca Augustar z. B. eine wichtige Etappe darstellt - immer unwichtiger zu werden; über Nachweise der Bücherstandorte $\mathrm{zu}$ verfügen, die offensichtlich oft verstreut sind, lässt den Wert der bibliographischen Karten 
immer deutlicher werden. Durch die elektronische Datenverarbeitung sind die Lückenhaftigkeit und das Ungenügen einzelner Bibliotheken deutlich hervorgetreten. Der Vergleich mit dem gesamten bibliothekarischen Erbe, von dem man Kenntnis haben kann, auch wenn er sich auf eine noch nicht vollständig elektronische Vergleichsgrundlage stützt, hat die dokumentarischen Sammlungen in den Käfig historischer Kontingenz und zufälliger geographischer Bestimmung gesperrt. Ein solches Resultat ist gleichbedeutend damit, metaphorisch gesprochen, dass man die Barrieren und militärischen Anlagen der Antike von einem darüber fliegenden Flugzeug aus wahrnimmt - ein neuerlicher Beweis dafür, dass die Überschreitung einer bestimmten Grenze oder einer bestimmten Dimension eine substanziale Modifikation in den Erfordernissen und den Bedingungen der Kommunikationssysteme provoziert. Wenn wir eine zusammenfassende Feststellung über die Anwesenheit oder Abwesenheit von Büchern versuchen, dann zeigen die elektronischen Erkundungen auf alarmierende Weise die Seltenheit, die Knappheit und die Qualität einzelner bibliothekarischer Sammlungen, wie reich sie auch immer sind; das verdeutlicht nicht nur die Grenzen und Unzulänglichkeiten der Bibliotheken, sondern auch, ganz dringend und immer wieder, die Kompetenzen, das Urteilsvermögen und die Kriterien der Bewertung auszubilden, die für die Wissenschaft und die bibliographische Ordnung charakteristisch sind. Es folgt daraus auch, dass Umfragen und Erhebungen sowie Vergleich der Bibliotheken untereinander mehr als je erfordern, dass sich ein bibliographisches Szenario etabliert, in welchem es möglich wäre, alle Bibliotheken mit all ihren Abteilungen zusammenzufassen. Die Bibliotheken waren früher reich, groß oder bescheiden; heute erscheinen sie, angesichts der Kenntnis, die man über ihre bibliographische Disponibilität hat, alle mehr oder weniger mangelhaft und voller Lücken.

Schließlich ist der Status der Virtualität bibliothekarischer Informationen - abgelöst von gelegentlicher oder zufälliger physischer Präsenz - ein Beitrag zur Etablierung der Priorität bibliographischer Strukturen und Informationen und folglich ein nachdrücklicher Aufruf dazu, sich rasch, mit Entschlossenheit, großer Offenheit und stringenter Kohärenz den Problemen ihrer Organisation auf den verschiedenen Ebenen dokumentarischer Nützlichkeit auszusetzen.

Es reicht allerdings nicht, das bestehende schriftliche Erbe aufzulisten, wenn man nicht zugleich die Sammlung in einem entsprechenden geeigneten Rahmen mit Respekt vor den Differenzen und der Komplexität realisiert. Dazu ist es notwendig, auch die Erwartungen und Anforderungen in ihrem ganzen Umfang zu betrachten, sowohl derjenigen, welche jene Dokumente hervorgebracht haben, wie auch die von denjenigen, die sie benutzen wollen.

\section{Plädoyer für eine Revidierung der Katalogisierungsverfahren}

Um noch direkter auf mein Thema einzugehen, möchte ich sagen - mit Absicht auf die Aufrechterhaltung einer bedeutsamen und fruchtbaren Korrelation zwischen der Realität der Dokumente und der Realität der Erkenntnis und der Kultur, in Harmonie und Kohärenz mit dem, was ich bis hierher gesagt habe -, dass man eher als die Stabilisierung der Beziehung zwischen Distribution und Anordnung der Bände in einer Bibliothek auf die Grade und die Qualität der Optionen und der Auswahlvorgänge, die die Wahl ihrer Bestandteile und ihrer bibliographische Komposition bestimmt haben, achten sollte.

Zwischen der Scylla des Informationstechnizismus und der Charybdis der literarischen, philologischen und historischen Instrumentalität schwankt das Schiffchen der Bibliographie also auf ungewissen und gefährlichen Gewässern. Die Geschichte der Bibliotheken - zumindest dann, wenn sie nicht zur Historiographie einer zweiten Natur degenerieren will - hat unvermeidlicherweise denselben Charakter und das Schicksal der Bibliographie, weil es keine Bibliothek ohne Organisation der Bücher gibt und keine Katalogisierung, die nicht von der bibliographischen Logik animiert und legitimiert wird.

Konrad Gessner war der erste, welcher die organisatorischen Prinzipien und die Erstellungstechniken der Kataloge theoretisch behandelt und angewendet hat, sowohl bibliothekarische als auch bibliographische Prinzipien im Innern eines Informations- und Verweisungssystems, das ex principio die Totalität der Wissenschaft, der Literatur und der gelehrten Philologie einschloss. Wenn wir uns dieses Beispiel vor Augen halten, sollten wir in einer analogen Vision der Allgemeinheit und des Respekts gegenüber der gesamten schriftlichen Tradition Geist, Energie und Durchhaltekraft wiederfinden.

Wenn die Kataloge als zusammenfassende Schemata, als umfassende Bilder oder repräsentative Symbole, als intime und allgemeine Signaturen des Wissens und der kognitiven Realität einer Epoche fungieren, können sie dies nur, indem sie die Ideale und die Utopien, die Taxonomien und Anordnungen, die wissenschaftlichen, technologischen, produktionsmäßigen, sozialen etc. Begriffe mit Bezug 
auf eine logisch-noetische Metastruktur interpretieren, welche eben die Bibliographie darstellt.

Um die Bilder und Repräsentationen einer Realität der Bücher zu bewerten, müssen die Bibliothekskataloge und die Bibliographien unleugbar das Wissen und die Bildung der in ihnen zusammengefassten Zeit widerspiegeln; ein abstrakter Isomorphismus zwischen der Wissenschaft und den Texten einer Epoche, den damals publizierten Büchern, dem Studium dieser Bücher und den Methoden ihrer Erschließung und Erforschung konstituiert die Gesamtheit der Bedingungen, um die Prozesse der schriftlichen Kommunikation $\mathrm{zu}$ individuieren und zu vernetzen. Unter der scheinbar ruhigen und geistlosen Oberfläche der Katalogisierungsverfahren gibt es die Auseinandersetzung von Formen und Modalitäten, welche die Instrumente bereitstellen, archivieren und benutzen, die die Organisation und den Zugang zum Bestand und zum aktiven Gedächtnis des schriftlichen Erbes der ganzen Menschheit garantieren.

Aber die Kataloge sind nicht nur einfach als Symbol mit Rücksicht auf eine Synthese vorangegangener Bedingungen zu bewerten; sie gehören auch auf ein logisches und ideologisches Meta-Niveau; die Situationen und die Charaktere dieses Meta-Niveaus wahrzunehmen und $\mathrm{zu}$ interpretieren zeigt mehr als die bloße Anwendung von vereinfachten und auf die unleugbar nur technische Natur der Katalogisierung bezogenen Meinungen. Mit der letzten Behauptung möchte ich nicht den Sinn des Titels dieses Vortrages offen widerrufen, sondern ihn ausdehnen und vertiefen. Um jedenfalls die Substanz zu verstehen, ist es notwendig, die Begriffe zu revidieren, die bis heute allgemein unkritisch gebraucht wurden, in Bezug auf die Ontologie und die Funktion der Bibliographie, der Katalogisierung und der Bibliothek, einschließlich der Beziehungen zur Geschichte der Bibliotheken.
Wenn die Paradigmen einer Tradition fallen, befindet man sich nicht nur in einem Zustand der Auflösung und der Unsicherheit, sondern es wird auch immer schwieriger, Beziehungen mit der Vergangenheit zu knüpfen, wie auch Ordnung in der Wissenssphäre zu setzen.

Eine der Wirkungen, als Beispiel für die gegenwärtige begriffliche Bedeutungsverschiebung ist die Tatsache, dass die Bibliotheken Opfer eines informationalen Kurzschlusses sind. Die Nachrichten und Botschaften gehen direkt, statt über das Mittel der dauerhaften Gestalt der Bücher, vom Produzenten zum Benutzer, mit dem Ergebnis, dass sich die Gelegenheiten verlieren, worin sich Orte und geistige Formen bilden, die in einer kommunikationellen Verschiebung die Möglichkeit geben, eine unverzichtbare kognitive Synthese zu errichten, die nicht allein zur Kohärenz und im existenziellen Gleichgewicht notwendig wäre, sondern auch zur Energieerschaffung und Impulsgebung für höhere geistige Ebenen diente.

Abschließend möchte ich betonen, dass jetzt, in weiterer und kritischer, spekulativer oder hermeneutischer Hinsicht, der Moment einer Überprüfung derjenigen Kategorien erreicht ist, welche im gesamten System der Kommunikationsverfahren zur Erfassung oder Abweichung impliziert sind, mit dem Ziel, dem Bereich der bibliographischen Disziplinen unter anderem eine strengere und produktivere Konfiguration zuzuweisen, die nicht nur für die wissenschaftliche Rahmung der entsprechenden Gegenstände und Funktionen nützlich ist, sondern auch für die effektive Verbesserung der Indizierungs- und Anordnungssysteme.

Aus dem Italienischen von Ulrich Johannes Schneider, mit Unterstützung von Francesco Tommasi und Patricia Carmassi. 